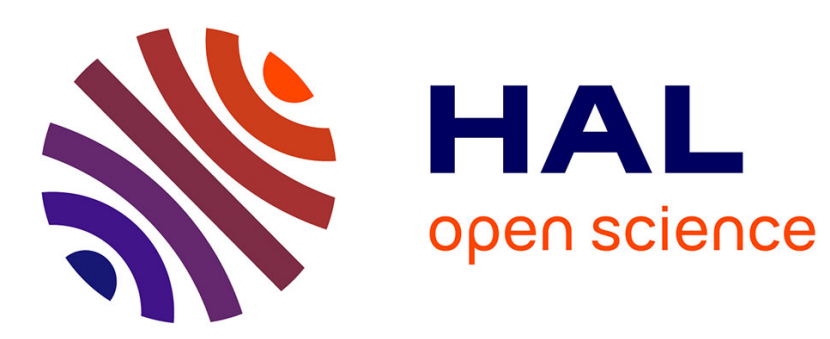

\title{
Effets thermiques en cavitation
}

Christian Pellone, Jean-Pierre Franc

\section{To cite this version:}

Christian Pellone, Jean-Pierre Franc. Effets thermiques en cavitation. European Journal of Environmental and Civil Engineering, 2008, 12 (5), pp.497-508. 10.1080/19648189.2008.9693026 . hal00936655

\section{HAL Id: hal-00936655 \\ https://hal.science/hal-00936655}

Submitted on 29 Oct 2020

HAL is a multi-disciplinary open access archive for the deposit and dissemination of scientific research documents, whether they are published or not. The documents may come from teaching and research institutions in France or abroad, or from public or private research centers.
L'archive ouverte pluridisciplinaire HAL, est destinée au dépôt et à la diffusion de documents scientifiques de niveau recherche, publiés ou non, émanant des établissements d'enseignement et de recherche français ou étrangers, des laboratoires publics ou privés. 


\title{
Effets thermiques en cavitation
}

\section{Christian Pellone - Jean-Pierre Franc}

\author{
LEGI \\ (Laboratoire des Ecoulements Géophysiques et Industriels) \\ INPG - UJF - CNRS \\ BP 53, F-38041 Grenoble cedex 09 \\ \{Christian.Pellone, Jean-Pierre.Franc\}@hmg.inpg.fr
}

\begin{abstract}
RÉSUMÉ. Un modèle simple basé sur la résolution de l'équation de Rayleigh décrivant l'évolution d'une bulle sphérique isolée est développé pour analyser les effets thermiques en cavitation. Deux approches sont utilisées pour modéliser le transfert de chaleur à travers l'interface liquide-vapeur. La première est basée sur un modèle de type convectif, la seconde sur la résolution de l'équation de diffusion de la chaleur dans le liquide entourant la bulle. Les deux modèles sont appliqués à un inducteur cavitant; les formes de cavités ainsi que les distributions de température sont comparées. Les évolutions de la longueur de cavité en fonction du nombre de cavitation pour l'eau froide (sans effet thermique) et pour le fluide réfrigérant $R 114$ à deux températures différentes sont confrontées aux résultats expérimentaux.
\end{abstract}

ABSTRACT. A simple model based on the resolution of Rayleigh equation for a single spherical bubble is used to analyse thermal effects in cavitation. Two different assumptions are considered for modelling heat transfer through the liquid-vapour interface. One is based upon a convective type approach, the other one upon the resolution of the heat diffusion equation in the liquid surrounding the bubble. Both models are applied to a cavitating inducer and cavity shapes and temperature distributions are compared. The evolution of cavity length with the cavitation number for cold water (without thermal effects) and for refrigerant $R 114$ at two different temperatures are compared to experimental data.

MOTS-CLÉS : cavitation, effet thermodynamique, équation de Rayleigh-Plesset, inducteur.

KEYWORDS: cavitation, thermodynamic effect, Rayleigh-Plesset equation, inducer. 


\section{Introduction}

L'effet thermodynamique en cavitation est caractérisé par un écart de température entre le liquide à l'infini et les zones diphasiques. Cet effet est dû à la chaleur latente de vaporisation qui, lors de la phase de vaporisation, est soutirée au liquide entourant la zone de cavitation dont la température se trouve alors abaissée. S'il est négligeable pour l'eau à température ambiante, il n'en est pas de même pour les fluides thermosensibles tels que les fluides cryogéniques utilisés pour la propulsion des moteurs-fusées.

Le refroidissement d'une cavité est caractérisé par le paramètre adimensionnel de (Stepanoff, 1964) défini par $\mathrm{B}=\left(\mathrm{T}_{\infty}-\mathrm{T}_{\mathrm{c}}\right) / \Delta \mathrm{T} *$. Il représente le rapport de l'écart de température $\Delta \mathrm{T}=\mathrm{T}_{\infty}-\mathrm{T}_{\mathrm{c}}$ entre la température du liquide loin de la cavité $\mathrm{T}_{\infty}$ et celle de la cavité $\mathrm{T}_{\mathrm{c}}$ à un écart caractéristique $\Delta \mathrm{T}^{*}$ défini par $\Delta \mathrm{T}^{*}=\rho_{\mathrm{v}} \mathrm{L} / \rho_{\ell} \mathrm{c}_{\mathrm{p} \ell}$ où $\rho_{\mathrm{v}}$ et $\rho_{\ell}$ sont les masses volumiques de la vapeur et du liquide et $\mathrm{L}$ et $\mathrm{c}_{\mathrm{p} \ell}$ la chaleur latente de vaporisation et la capacité calorifique du liquide. $\Delta \mathrm{T}^{*}$ est le refroidissement qu'il faudrait appliquer à une unité de volume de liquide pour recueillir la chaleur latente nécessaire à la vaporisation d'une unité de volume de vapeur. La valeur de ce paramètre dépend du fluide utilisé et constitue un indicateur de l'importance des effets thermiques en cavitation. Il est de l'ordre de $0,01 \mathrm{~K}$ pour l'eau à température ambiante alors qu'il vaut environ $1 \mathrm{~K}$ pour l'hydrogène liquide à $22 \mathrm{~K}$. L'importance des effets thermiques peut également être mesurée par le paramètre de (Brennen, 1995) $\Sigma=\Delta \mathrm{p}_{\mathrm{v}}^{*} /\left(\rho_{\ell} \sqrt{\alpha_{\ell}}\right)$ ou de façon équivalente par le paramètre de (Kato, 1984) $\alpha=\Sigma \sqrt{\rho_{\ell} / \rho_{\mathrm{v}}}, \Delta \mathrm{p}_{\mathrm{v}}{ }^{*}$ étant l'écart de pression correspondant à $\Delta \mathrm{T}^{*}$ et défini par $\Delta \mathrm{p}_{\mathrm{v}}^{*}=\Delta \mathrm{T}^{*} \mathrm{dp}_{\mathrm{v}} / \mathrm{dT}, \mathrm{dp}_{\mathrm{v}} / \mathrm{dT}$ étant la pente de la courbe de pression de vapeur $\mathrm{p}_{\mathrm{v}}$ et $\alpha_{\ell}$ la diffusivité thermique du liquide.

Dans les modèles à interface pour lesquels la frontière entre le liquide et la vapeur (qu'il s'agisse d'une bulle ou d'une cavité) est bien définie, le transfert de chaleur par la seule diffusivité thermique moléculaire du liquide est largement insuffisant pour rendre compte des ordres de grandeur de refroidissement mesurés. Pour obtenir une prédiction correcte, (Kato, 1984; Watanabe et al., 2005) ont montré qu'elle devait être multipliée par un coefficient d'amplification $\varepsilon$ pouvant aller jusqu'à $10^{5}$. Cette valeur élevée est généralement attribuée à la turbulence qui améliore considérablement l'efficacité des transferts thermiques. Cette tendance a été confirmée par (Fruman et al., 1999) sur la base du modèle d'entraînement (Billet et al., 1981).

Parallèlement aux modèles à interface, des modèles diphasiques ont été développés. Le mélange des deux phases est en général considéré comme un pseudofluide homogène obéissant aux équations de Navier-Stokes. Dans ce cas, une loi d'état doit être introduite pour le mélange, par exemple une loi barotrope (Rapposelli et al., 2003 ; Rolland et al., 2006). Une autre approche consiste à résoudre une 
équation de transport supplémentaire pour la phase vapeur comportant un terme source modélisant le phénomène de cavitation (Tani et al., 2002; Hosangadi et al., 2005). Le processus de vaporisation ou de condensation peut être modélisé par des équations spécifiques (Hosangadi et al., 2005) ou en ensemençant le fluide en microbulles et en suivant leur évolution grâce à l'équation de Rayleigh-Plesset (Kubota et al., 1992 ; Singhal et al., 2002). Pour tenir compte des effets thermiques, il est alors nécessaire d'adjoindre une équation d'énergie aux équations de conservation de masse et de quantité de mouvement du mélange.

Dans le cas des modèles à interface, le volume de liquide entourant la vapeur étant quasi infini, le transfert de chaleur est limité par le taux de croissance de la couche limite thermique. Par contre, les modèles diphasiques qui font l'hypothèse d'un mélange intime entre le liquide et sa vapeur sont tels que le volume de liquide entourant une bulle est naturellement limité par les bulles environnantes. De fait, pour les modèles diphasiques, les effets thermiques sont principalement limités par le volume de liquide capable de fournir la chaleur de vaporisation et non par la croissance d'une couche limite thermique. Les effets thermiques sont alors contrôlés par l'évolution du taux de vide et ne dépendent pas directement de la diffusivité thermique du liquide.

La différence entre les deux types de modèles est confirmée en comparant les expressions du facteur B. Dans le cas d'une bulle qui passe d'un rayon nul à un rayon $\mathrm{R}$ pendant le temps t, le facteur $\mathrm{B}$ est donné par $\mathrm{B} \approx \mathrm{R} / \sqrt{\alpha_{\ell} \mathrm{t}}$ (Franc et al., 2004a). Pour un mélange diphasique de taux de vide $\alpha_{v}$, la conservation de la chaleur conduit à la relation $\mathrm{B} \approx \alpha_{\mathrm{v}} /\left(1-\alpha_{\mathrm{v}}\right)$ (Franc et al., 2004b). Ces relations montrent clairement que les effets thermiques pour une bulle isolée dépendent de la diffusivité thermique du liquide, ce qui n'est pas le cas pour le modèle diphasique. De plus, le facteur B devient singulier quand le taux de vide tend vers 1, ce qui montre que le modèle diphasique n'est plus valable quand la cavité n'est composée que de vapeur. Il est donc important en pratique de reconnaître le type de cavitation afin d'identifier le mécanisme physique qui limite l'apport de chaleur nécessaire à la vaporisation.

Cet article est consacré au développement d'une méthode d'analyse des effets thermiques en cavitation basée sur la résolution de l'équation de Rayleigh pour la dynamique d'une bulle de vapeur. Il appartient à la classe des modèles à interface pour lesquels le transfert de chaleur au travers de la couche limite thermique est le phénomène physique limitatif. L'originalité de cette contribution réside dans le fait qu' un terme relatif aux effets thermiques est introduit dans l'équation de Rayleigh et que deux approches, l'une convective et l'autre conductive, sont considérées pour expliciter ce terme. Les résultats de la modélisation sont comparés à des résultats d'essais d'un inducteur de turbopompe spatiale (Franc et al., 2004b) obtenus en fluide réfrigérant de type R114 connu pour présenter des effets thermiques relativement importants. 
Le calcul est mené en deux étapes : la première consiste à déterminer le champ de pression. Pour cela, une méthode intégrale aux frontières a été utilisée; la seconde consiste à résoudre l'équation de Rayleigh en utilisant la distribution de pression calculée précédemment. La forme de la cavité est assimilée à l'enveloppe d'une bulle hémisphérique qui grossit puis implose sur l'extrados de la pale. Ce genre de modèle présente un certain nombre de limitations. L'une d'entre elles est que le champ de pression n'interagit pas avec la cavité puisque la bulle est calculée à partir d'un champ de pression obtenu en écoulement non cavitant. La nature 2D du calcul en est une autre puisque les inducteurs peuvent être le siège d'écoulements de retour pouvant changer de façon significative les angles d'attaque. Néanmoins, cette contribution fournit un support théorique utile à l'interprétation et à la compréhension physique de résultats d'essais.

\section{Formulation et résolution}

\subsection{Calcul de l'écoulement subcavitant de base}

L'inducteur est représenté par une grille d'aubes supposées planes (figure 1). Cette configuration 2D est classiquement obtenue par une section cylindrique à un rayon $r$ donné de la machine tournante. L'épaisseur relative des pales est de l'ordre de $1 \%$ et la solidité $\mathrm{c} / \mathrm{H}$ vaut 2 . La vitesse d'entrée $\mathrm{V}_{\mathrm{u}} \overrightarrow{\mathrm{i}}$ est supposée perpendiculaire à la grille (pas de prérotation). A l'infini amont, la vitesse relative de référence est $\mathrm{W}_{\mathrm{u}}=\sqrt{\mathrm{V}_{\mathrm{u}}^{2}+\omega^{2} \mathrm{r}^{2}}$ ( $\omega$ vitesse angulaire de rotation). L'écoulement radial normal à la surface cylindrique est ignoré et l'écoulement est supposé bidimensionnel dans le plan $(\vec{i}, \vec{j})$.

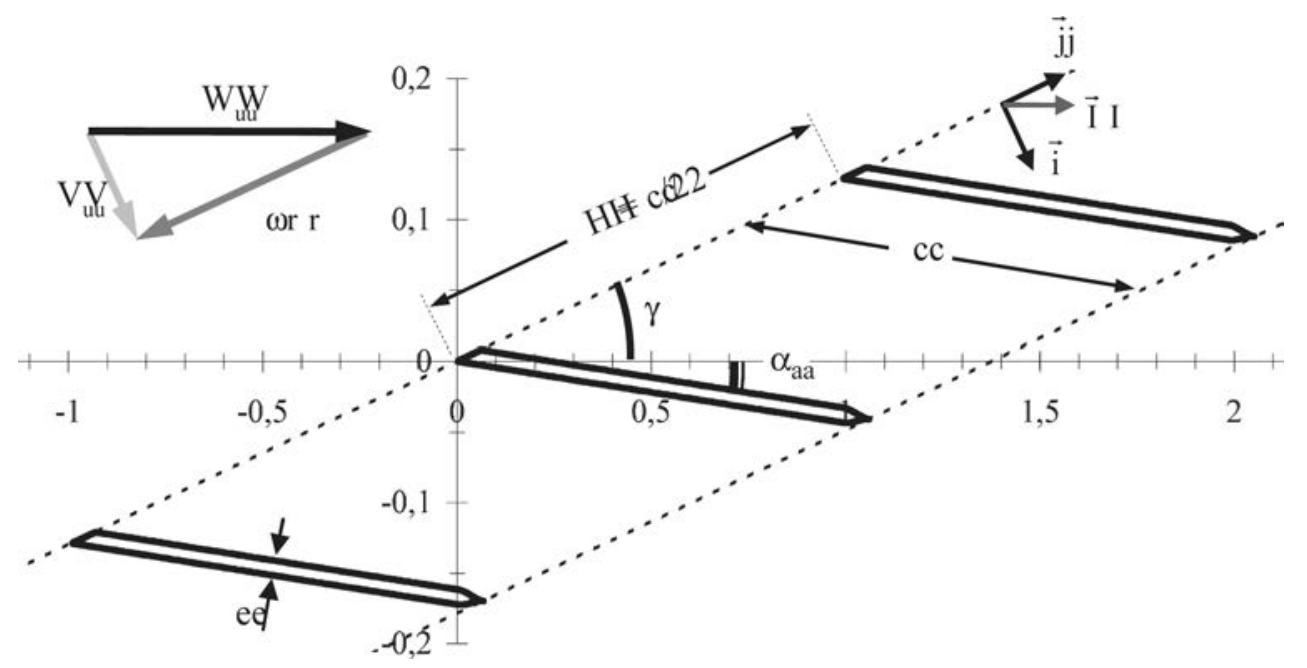

Figure 1. Schéma de la grille d'aubes (échelle verticale dilatée) 
La distribution de pression sur la pale est calculée par la méthode des équations intégrales aux frontières. Une double distribution de sources et de tourbillons est répartie sur la surface d'une pale avec une périodicité $\mathrm{H}$ suivant la direction de la grille afin de prendre en compte la série infinie de pales. Elle est choisie de façon à ce que la vitesse relative à l'infini amont soit dans la direction $\overrightarrow{\mathrm{I}}$. Les conditions aux limites sont une condition de glissement sur la pale et la continuité de pression au bord de fuite. La conservation du débit dans l'inducteur nécessite que la composante normale au front de grille de la vitesse relative soit la même à l'infini amont et aval. La double distribution de singularités est calculée de façon à minimiser les erreurs dues à la discrétisation.

\subsection{Equation de Rayleigh avec effets thermiques}

En négligeant les effets visqueux, la tension superficielle à l'interface et les effets de gaz non condensables, ce qui est justifié dès que le germe de cavitation devient une bulle macroscopique, l'évolution au cours du temps t d'une bulle sphérique de rayon $\mathrm{R}$ en milieu infini est donnée par l'équation classique de Rayleigh :

$$
\mathrm{R} \frac{\mathrm{d}^{2} \mathrm{R}}{\mathrm{dt}^{2}}+\frac{3}{2}\left(\frac{\mathrm{dR}}{\mathrm{dt}}\right)^{2}=\frac{\mathrm{p}_{\mathrm{v}}\left(\mathrm{T}_{\mathrm{c}}\right)-\mathrm{p}(\mathrm{t})}{\rho_{\ell}}
$$

Le champ de pression $\mathrm{p}(\mathrm{t})$ est celui auquel la bulle est assujettie lorsqu'elle se déplace sur l'extrados de la pale. Il résulte du calcul présenté à la section précédente. Pour prendre en compte les effets thermiques dans l'équation [1], il est nécessaire de considérer la pression de vapeur $\mathrm{p}_{\mathrm{v}}$ à la température de cavité $\mathrm{T}_{\mathrm{c}}$ qui est différente de la température $\mathrm{T}_{\infty} \mathrm{du}$ liquide à l'infini. En désignant par $\mathrm{p}_{\mathrm{u}}$ la pression à l'infini amont, on définit le coefficient de pression par $\mathrm{C}_{\mathrm{p}}=2\left(\mathrm{p}-\mathrm{p}_{\mathrm{u}}\right) /\left(\rho_{\ell} \mathrm{W}_{\mathrm{u}}^{2}\right)$ et le paramètre de cavitation par $\sigma_{\mathrm{v}}=2\left(\mathrm{p}_{\mathrm{u}}-\mathrm{p}_{\mathrm{v}}\left(\mathrm{T}_{\infty}\right)\right) /\left(\rho_{\ell} \mathrm{W}_{\mathrm{u}}^{2}\right)$. En supposant que la bulle se déplace sur la pale avec la vitesse locale du fluide $\mathrm{W}_{\mathrm{u}} \sqrt{1-\mathrm{C}_{\mathrm{p}}}$, on peut transformer les dérivées en temps en dérivées spatiales par la relation $\mathrm{d} / \mathrm{dt}=\mathrm{W}_{\mathrm{u}} \sqrt{1-\mathrm{C}_{\mathrm{p}}} \mathrm{d} / \mathrm{dx}, \mathrm{x}$ désignant l'abscisse curviligne sur la pale. En adimensionnalisant les variables spatiales par la corde c de la pale (variables surlignées) et en désignant par $\bullet$ la dérivée par rapport à $\bar{x}=\mathrm{x} / \mathrm{c}$, on obtient la forme sans dimension suivante de l'équation [1] :

$$
\left[1-\mathrm{C}_{\mathrm{p}}\right]\left[\overline{\mathrm{R}} \ddot{\overline{\mathrm{R}}}+\frac{3}{2} \dot{\mathrm{R}}^{2}\right]-\frac{1}{2} \dot{\mathrm{C}_{\mathrm{p}}} \overline{\mathrm{R}} \dot{\overline{\mathrm{R}}}+\frac{1}{\rho_{\ell} \mathrm{W}_{\mathrm{u}}^{2}} \frac{\mathrm{dp}_{\mathrm{v}}}{\mathrm{dT}}\left(\mathrm{T}_{\infty}-\mathrm{T}_{\mathrm{c}}\right)=-\frac{\mathrm{C}_{\mathrm{p}}+\sigma_{\mathrm{v}}}{2}
$$

Deux modèles sont utilisés pour expliciter le troisième terme du membre de gauche décrivant les effets thermiques. 
Cette approche suppose que le transfert de chaleur à l'interface est proportionnel à la différence de température, c'est-à-dire de la forme $\mathrm{h}\left(\mathrm{T}_{\infty}-\mathrm{T}_{\mathrm{c}}\right)$. En l'absence de corrélation validée pour les écoulements cavitants, le coefficient de transfert de chaleur convectif h est considéré ici comme un paramètre d'ajustement. Le bilan de chaleur à l'interface de la bulle s'écrit sous la forme $L d\left(4 / 3 \pi R^{3} \rho_{v}\right) / d t=4 \pi R^{2} h\left(T_{\infty}-T_{c}\right)$, où $L$ désigne la chaleur latente de vaporisation. Il permet d'expliciter la différence de température, qui apparait proportionnelle au taux de variation du rayon de bulle :

$$
\Delta \mathrm{T}=\mathrm{T}_{\infty}-\mathrm{T}_{\mathrm{c}}=\frac{\rho_{\mathrm{v}} \mathrm{L}}{\mathrm{h}} \frac{\mathrm{dR}}{\mathrm{dt}}
$$

Pendant la phase de croissance, la température de la bulle est donc inférieure à la température du liquide et inversement pendant la phase d'implosion. En reportant l'expression [3] dans l'équation générale [2], on obtient l'équation de Rayleigh pour l'approche convective :

$$
\left[1-\mathrm{C}_{\mathrm{p}}\right]\left[\overline{\mathrm{R}} \ddot{\overline{\mathrm{R}}}+\frac{3}{2} \dot{\mathrm{R}}^{2}\right]-\frac{1}{2} \dot{\mathrm{C}}_{\mathrm{p}} \overline{\mathrm{R}} \dot{\mathrm{R}}+\frac{1}{\mathrm{~N}_{\mathrm{u}}} \frac{\Delta \mathrm{p}_{\mathrm{v}}^{*}}{\rho_{\ell} \alpha_{\ell}} \tau \sqrt{1-\mathrm{C}_{\mathrm{p}}} \dot{\overline{\mathrm{R}}}=-\frac{\mathrm{C}_{\mathrm{p}}+\sigma_{\mathrm{v}}}{2}
$$

où $\mathrm{N}_{\mathrm{u}}=\mathrm{h} \mathrm{c} / \lambda_{\ell}$ est le nombre de Nusselt et $\tau=\mathrm{c} / \mathrm{W}_{\mathrm{u}}$ le temps caractéristique de transit $\left(\lambda_{\ell}\right.$ : conductivité thermique du liquide). L'intensité des effets thermiques dépend $\mathrm{du}$ rapport $\tau / \tau_{\mathrm{T}}$, où $\tau_{\mathrm{T}}=\mathrm{N}_{\mathrm{u}}\left(\rho_{\ell} \alpha_{\ell}\right) / \Delta \mathrm{p}_{\mathrm{v}}^{*}$ peut être considéré comme un temps thermique puisqu'il contient l'information sur le transfert de chaleur à l'interface. Si ce rapport est petit devant 1, les effets thermiques sont négligeables et on retrouve l'équation classique de Rayleigh. Outre le nombre de Nusselt, le temps thermique dépend du facteur $\Delta \mathrm{p}_{\mathrm{v}}^{*} /\left(\rho_{\ell} \alpha_{\ell}\right)$ dont l'expression est très proche de celle du paramètre de Brennen.

\subsubsection{Approche conductive}

Cette seconde approche est basée sur la résolution de l'équation de diffusion de la chaleur dans le liquide entourant la bulle. En remarquant que l'épaisseur de la couche limite thermique est généralement beaucoup plus petite que le rayon de la bulle, (Zwick et Plesset, 1955) ont obtenu l'expression analytique suivante de la température de la bulle à l'interface : 


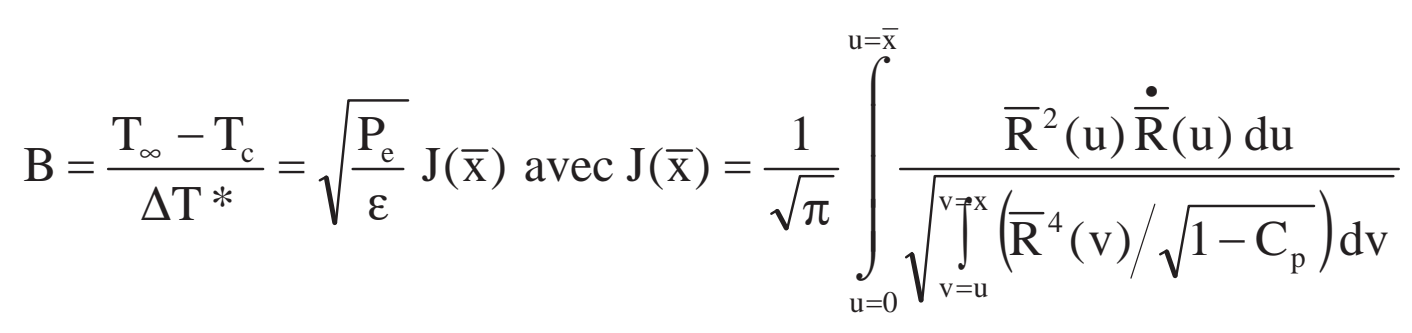

En la reportant dans l'équation générale [2] et en introduisant le paramètre de Brennen, on obtient l'équation de Rayleigh pour l'approche conductive qui, compte tenu de l'expression de $\mathrm{J}$ est une équation intégro-différentielle complexe :

$$
\left[1-\mathrm{C}_{\mathrm{p}}\right]\left[\overline{\mathrm{R}} \ddot{\overline{\mathrm{R}}}+\frac{3}{2} \dot{\mathrm{R}}^{2}\right]-\frac{1}{2} \dot{\mathrm{C}}_{\mathrm{p}} \overline{\mathrm{R}} \dot{\overline{\mathrm{R}}}+\Sigma \sqrt{\frac{\mathrm{c}}{\mathrm{W}_{\mathrm{u}}^{3}}} \frac{1}{\sqrt{\varepsilon}} \mathrm{J}=-\frac{\mathrm{C}_{\mathrm{p}}+\sigma_{\mathrm{v}}}{2}
$$

Dans certains cas, et particulièrement quand les effets thermiques sont importants, le modèle conductif peut conduire à des formes de cavité oscillantes irréalistes. Cet effet est observé dans la zone de fermeture de la cavité, là où la bulle collapse, c'est-à-dire quand la pression locale est a priori supérieure à la pression de vapeur. Si les effets thermiques sont importants, l'accroissement de température dû à la condensation augmente très sensiblement la pression de vapeur qui peut alors dépasser la pression locale. De ce fait, la dynamique de la bulle s'inverse et elle grossit à nouveau ce qui provoque l'oscillation de l'interface. Afin d'éviter cet effet, la différence de température $T_{c}-T_{\infty}$ est mise à zéro dès qu'elle devient positive, aussi bien pour le modèle conductif que pour le modèle convectif. Cela revient à supposer que la température de la bulle ne peut jamais dépasser la température du liquide ou, en d'autres termes, que la diffusion de la chaleur est considérablement accrue pendant la phase de collapse de sorte que la température s'uniformise presque instantanément. L'instabilité de la forme sphérique pendant le collapse et l'éclatement en petites structures de vapeur qui s'ensuit justifie cette hypothèse adoptée également par (Watanabe et al., 2005).

La résolution de l'équation de Rayleigh [4] ou [6] est faite à l'aide de deux méthodes de Runge-Kutta à pas adaptatif et couplées (Fortin, 2002).

\section{Résultats}

\subsection{Analyse comparative}

Les formes de cavité ainsi que les distributions de température obtenues par les deux modèles sont présentées figure 2. Les valeurs du nombre de Nusselt $\mathrm{N}_{\mathrm{u}}$ pour le modèle convectif et du coefficient $\varepsilon$ pour le modèle conductif ont été ajustées de telle façon que les longueurs de cavité soient identiques. Pour le modèle convectif, l'équation [3] montre que l'écart de température est proportionnel à la vitesse de l'interface. Celle-ci étant maximum au point de détachement de la cavité où la pression est minimum, l'écart de température est également maximum en ce point, ce 
qui explique la discontinuité de température observée au point de détachement. Pour le modèle conductif par contre, l'écart de température est nul au point de détachement avec un minimum au tiers de la longueur de cavité environ. Ce comportement plus régulier est une conséquence de la résolution de l'équation de diffusion de la chaleur au sein du liquide.

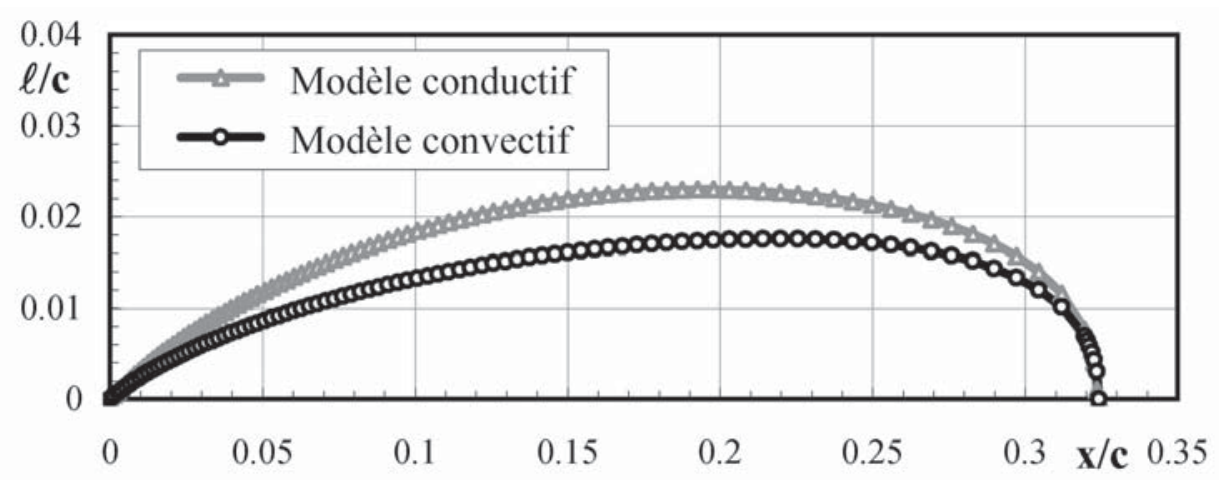

(a) forme de cavité

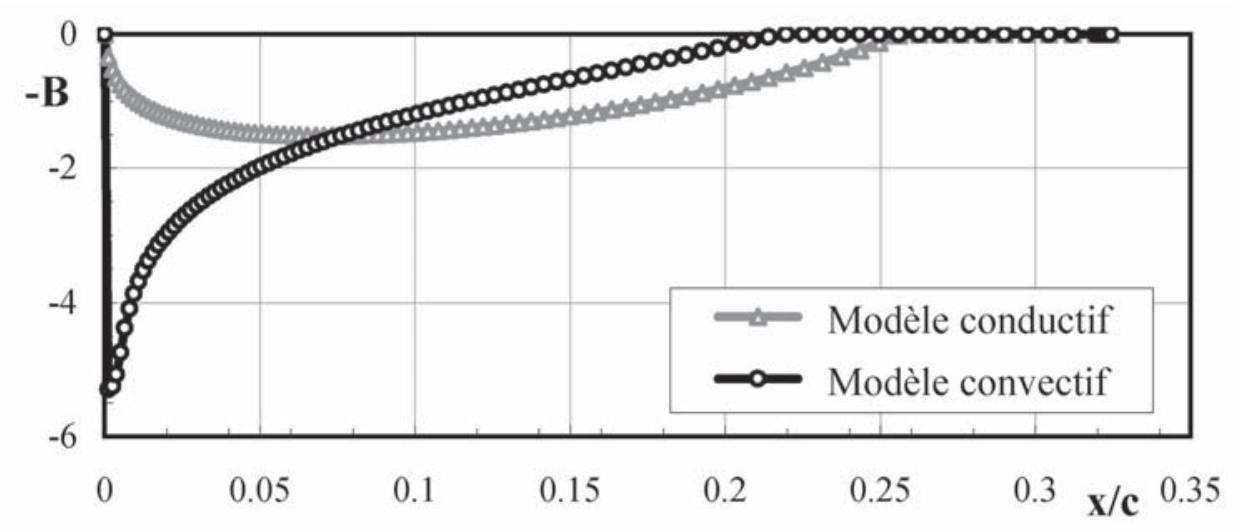

(b) température

Figure 2. Comparaison entre le modèle convectif $\left(N_{u}=7,3110^{6}\right)$ et le modèle conductif $\left(\varepsilon=510^{4}\right)$. Fluide R114 à $104,00^{\circ} \mathrm{F}$ et $\sigma_{v}=0$

Du fait de la discontinuité de température au départ de la cavité, la vitesse initiale de croissance de la bulle est plus petite pour l'approche convective que pour l'approche conductive pour laquelle elle est d'ailleurs la même qu'en l'absence d'effets thermiques vu que l'écart de température est initialement nul. Ceci explique pourquoi les cavités prédites par le modèle convectif sont plus minces. Par ailleurs, l'épaisseur maximum de cavité apparaît plus proche du point de fermeture pour le modèle convectif. Sa position correspond exactement au point où le facteur $B$ s'annule, toujours à cause de la proportionnalité entre $\mathrm{B}$ et la vitesse d'interface. Pour le modèle conductif, la relation [5] montre que le facteur B dépend de toute l'histoire de la température du liquide environnant. De fait, le point où l'épaisseur de cavité est maximum ne correspond plus à celui où le facteur B s'annule. 


\subsection{Influence des deux paramètres $N_{u}$ et $\varepsilon$}

Lorsque le nombre de Nusselt ou le paramètre $\varepsilon$ diminue, les transferts thermiques à l'interface deviennent moins efficaces et le retard d'origine thermique s'accroît. Il en résulte une diminution de la longueur de cavité comme le montre la figure 3, tendance qui est en accord avec l'expérience. Pour des effets thermiques très importants, c'est-à-dire pour des valeurs de $\mathrm{N}_{\mathrm{u}}$ ou de $\varepsilon$ en dessous d'un certain seuil, la longueur de cavité tend à se stabiliser à une valeur minimum non nulle. L'épaisseur de cavité ainsi que son volume tendent par contre vers zéro. La vitesse et l'accélération de l'interface deviennent négligeables et les termes inertiels du premier membre de l'équation de Rayleigh [1] aussi. Il en résulte que le second membre de [1] doit aussi s'annuler. En conséquence, quel que soit le modèle thermique utilisé, la pression de vapeur $\mathrm{p}_{\mathrm{v}}\left(\mathrm{T}_{\mathrm{c}}\right)$ évaluée à la température de cavité en chaque point doit tendre vers la distribution de pression sur la pale. A la limite, lorsque les effets thermiques sont largement prédominants et que le volume de la cavité devient très petit, la distribution de température dans la cavité est donc entièrement déterminée par la distribution de pression sur la pale et la loi de pression de vapeur du fluide.

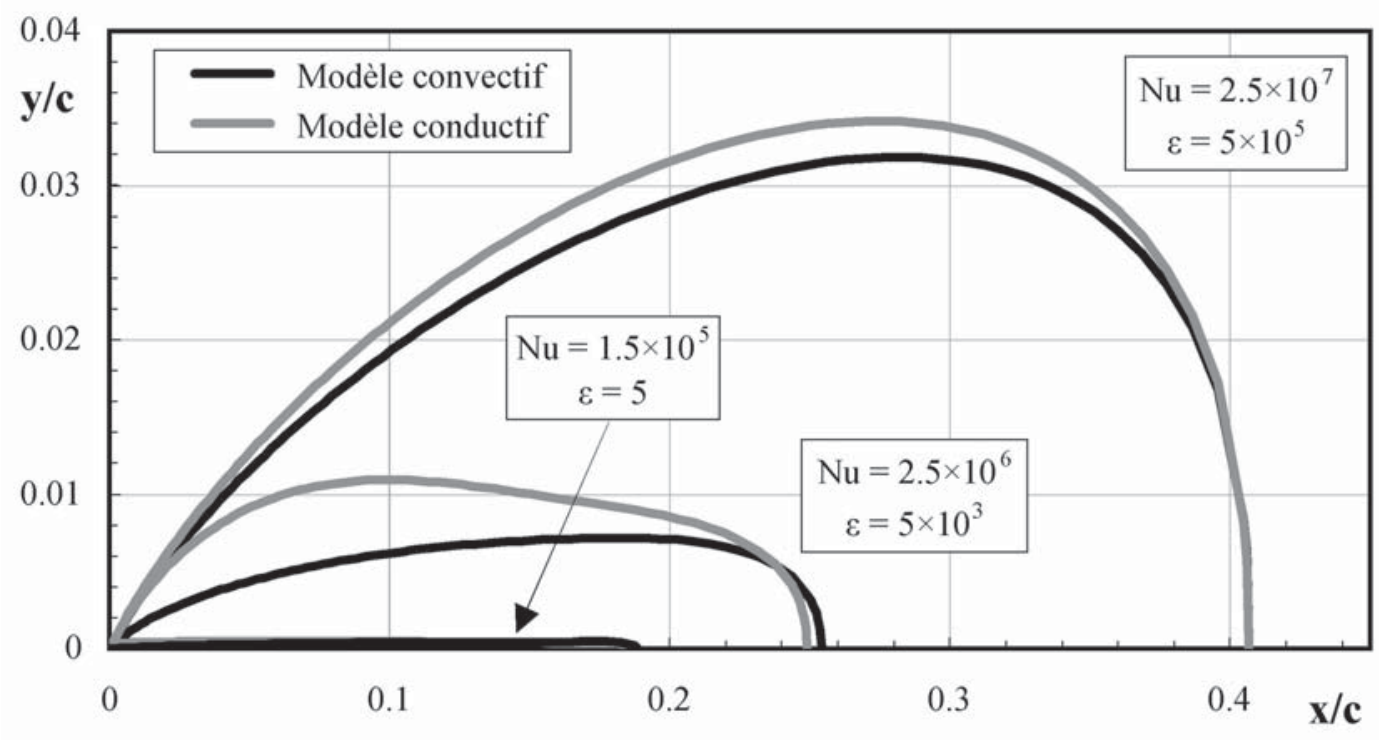

Figure 3. Influence de $N_{u}$ ou de $\varepsilon$ sur le développement de la cavitation. Fluide $R 114 \grave{a} 40^{\circ} \mathrm{C}$ et $\sigma_{v}=0$

\subsection{Comparaison avec les résultats expérimentaux}

La figure 4 présente l'évolution de la longueur de cavité en fonction du nombre de cavitation pour l'eau et le R114. Sur cette figure le nombre de cavitation est celui de l'inducteur défini par $\sigma_{\text {inducteur }} / \sigma_{\mathrm{v}} \cong \mathrm{r}^{2} / \mathrm{R}_{\text {ind }}^{2}$, où $\mathrm{R}_{\text {ind }}$ est le rayon périphérique de l'inducteur. Dans le cas de l'eau, c'est-à-dire sans effet thermique, la courbe calculée $\ell(\sigma)$ est en accord satisfaisant avec l'expérience même si des écarts sont 
observés en particulier à faible $\sigma$ du fait probablement de l'approximation rudimentaire de la forme réelle de pale par une plaque plane, de l'absence de couplage entre l'écoulement de base et le développement de la cavitation et de l'absence de prise en compte des effets tridimensionnels.

Le calcul a été conduit avec $\varepsilon=5000$ pour le modèle conductif, $\mathrm{N}_{\mathrm{u}}=2,08510^{6}$ pour le convectif. Ces valeurs ont été ajustées de façon à ce que les deux modèles donnent la même longueur de cavité dans le cas particulier $\sigma=0$ pour le R114 à $20^{\circ} \mathrm{C}$ et que cette longueur commune soit par ailleurs proche de celle obtenue par l'expérience. Ces valeurs particulières du nombre de Nusselt $\mathrm{N}_{\mathrm{u}}$ et du paramètre $\varepsilon$ ont ensuite été gardées constantes dans tous les calculs y compris pour le R114 à $40{ }^{\circ} \mathrm{C}$. La comparaison entre l'expérience et le calcul montre que les deux modèles conduisent, lorsque la température augmente, à une réduction de la longueur de cavité du même ordre de grandeur que celle observée expérimentalement.

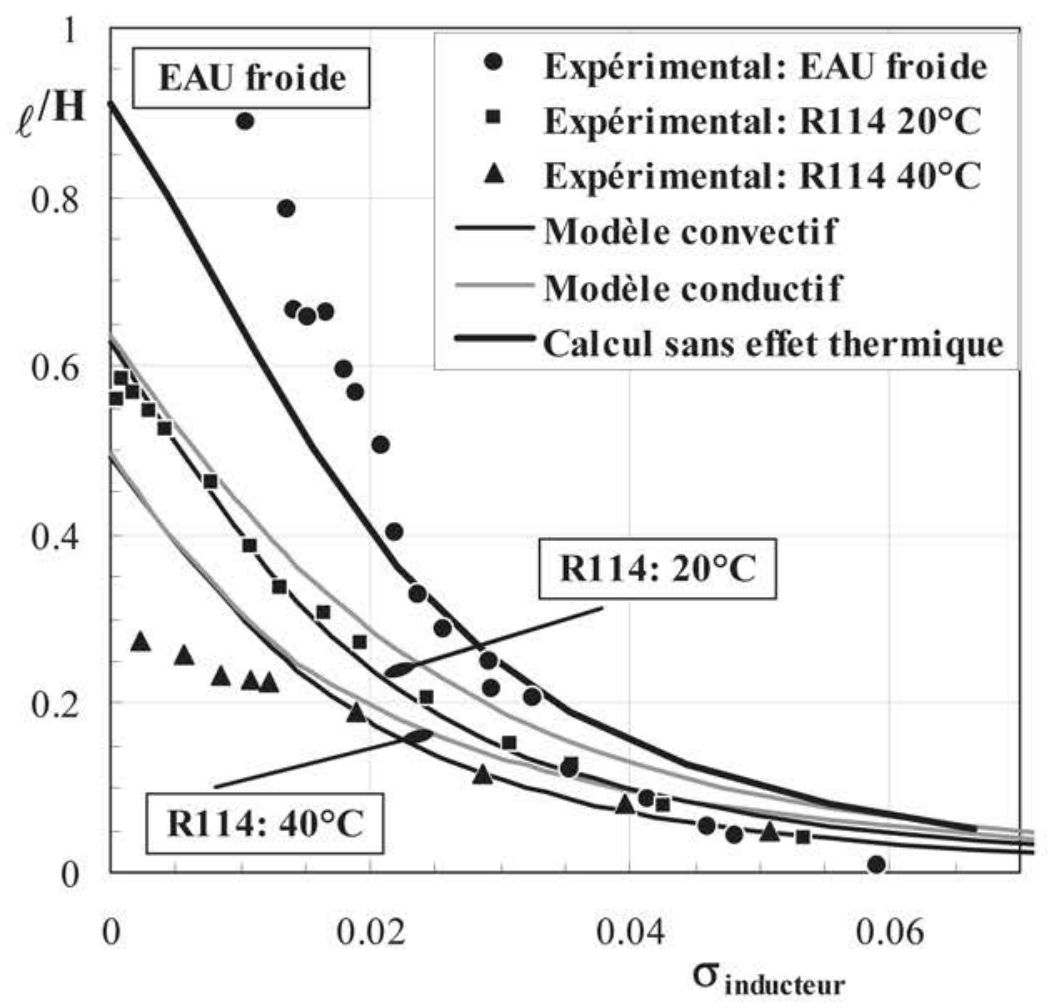

Figure 4. Longueur de cavité en fonction du nombre de cavitation. Les résultats expérimentaux sont extraits de (Franc et al., 2004)

La figure 5 présente une comparaison des formes de cavité obtenues avec le modèle conductif, en eau (sans effet thermique) et en $\mathrm{R} 114$ à $20^{\circ} \mathrm{C}$ et $40{ }^{\circ} \mathrm{C}$. La cavité devient de plus en plus mince quand les effets thermiques augmentent. Les formes de cavité avec et sans effet thermique ne sont pas en similitude géométrique. D'après l'équation [6], une similitude exacte nécessite la conservation du paramètre $\Sigma \sqrt{\mathrm{c} / \mathrm{W}_{\mathrm{u}}{ }^{3}}$ caractérisant les effets thermiques, en plus de la similitude habituelle en paramètre de cavitation $\sigma$. Les résultats ne peuvent être transposés rigoureusement 
que si ce paramètre additionnel est conservé. La même conclusion s'applique à la distribution de température de cavité qui ne peut pas être transposée simplement de l'eau au R114, ni entre deux températures différentes en R114, du fait de la distorsion introduite par le paramètre de similitude thermique supplémentaire.

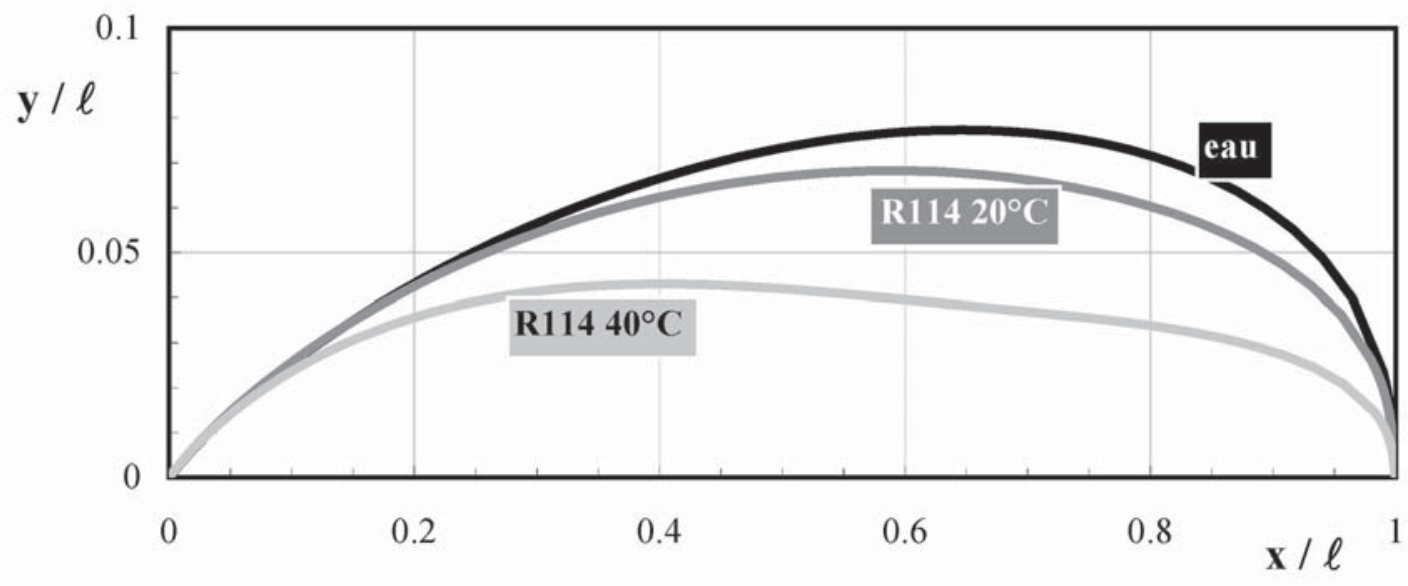

Figure 5. Influence de l'effet thermodynamique sur la forme de cavité. Longueur de cavité constante l/H $=0,5$ (modèle conductif $\varepsilon=5000$ )

\section{Conclusion}

La méthode d'analyse des effets thermiques présentée ici reproduit de façon satisfaisante les tendances globales sur l'évolution de la longueur de cavité en fonction du paramètre de cavitation, mises en évidence expérimentalement sur un inducteur cavitant, que le transfert thermique à l'interface soit supposé de nature convective ou conductive. L'approche convective semble moins réaliste du fait de la discontinuité de température qu'elle génère au point de détachement de la cavité. Bien que les résultats expérimentaux de (Hord, 1972 ; 1973 ; 1974 ; Fruman et al., 1999) tendent à montrer que le refroidissement est maximal juste à l'aval du point de détachement, il semblerait que l'extremum observé expérimentalement ne soit pas aussi marqué que celui prévu par l'approche convective, les profils de température mesurés étant généralement relativement plats. Le modèle conductif semblerait donc conduire à un comportement plus proche de celui révélé par l'expérience. Des développements complémentaires sont nécessaires pour d'une part, affiner la modélisation et, d'autre part, rendre la méthode prédictive, ce qui nécessiterait de corréler le nombre de Nusselt ou la diffusivité thermique turbulente aux caractéristiques générales de l'écoulement et en particulier aux nombres de Reynolds et de Prandtl sur la base de résultats expérimentaux. 
Cette étude a été menée grâce au soutien du CNES et de SNECMA, Direction moteurs spatiaux.

\section{Bibliographie}

Billet M. L., Holl J.W., Weir D. S., "Correlations of thermodynamic effects for developed cavitation", J. of Fluids Eng., vol. 103, December 1981, p. 534-542.

Brennen C. E., Cavitation and Bubble Dynamics, Oxford Univ. Press, 1995.

Fortin A., Analyse numérique, Presses internationales polytechniques, 2002.

Franc J. P., Michel J. M., Fundamentals of Cavitation, Kluwer Academic Publishers, 2004a.

Franc J. P., Rebattet C., Coulon A., "An experimental investigation of thermal effects in a cavitating inducer”, J. of Fluids Eng., vol. 126, September 2004b, p. 716-723.

Fruman D. H., Reboud J. L., Stutz B., "Estimation of thermal effects in cavitation of thermosensible liquids", Int. J. of Heat and Mass Transfer, vol. 42, 1999, p. 3195-3204.

Hord J., Cavitation in liquid cryogens, NASA reports CR-2054, CR-2156 (1972), CR-2242 (1973), CR-2448 (1974).

Hosangadi A., Ahuja V., "Numerical study of cavitation in cryogenic fluids", J. of Fluids Eng., vol. 127, March 2005, p. 267-281.

Kato H., "Thermodynamic effect on incipient and developed sheet cavitation", Int. Symp. on Cavitation inception, FED-vol.16, New-Orleans (USA), December 9-14, 1984, p. 127-136.

Kubota A., Kato H., Yamaguchi H., "A new modeling of cavitating flows: A numerical study of unsteady cavitation on a hydrofoil section", J. Fluid Mech., 240, 1992, p. 59-96.

Rapposelli E., d'Agostino L., "A barotropic cavitation model with thermodynamic effects", $5^{\text {th }}$ Int. Symp. on Cavitation, Osaka, Japan, November 1-4, 2003.

Rolland J., Boitel G., Barre S., Goncalves E., Fortes Patella R., "Experiments and modelling of cavitating flows in Venturi - Part I: stable cavitation", $6^{\text {th }}$ Int. Symp. on Cavitation, Wageningen, The Netherlands, September 2006.

Singhal A. K., Athavale M. M., Li H., Jiang Y., "Mathematical basis and validation of the full cavitation model", J. of Fluids Engineering, vol. 124, September 2002, p. 617-624.

Stepanoff A. J., "Cavitation properties of liquids", J. of Eng. for Power, April 1964, p. 195-200.

Tani N., Nagashima T., "Numerical analysis of cryogenic cavitating flow on hydrofoil - Comparison between water and cryogenic fluids", $4^{\text {th }}$ Int. Conf. on Launcher Technology, Liege (Belgium), Dec. 3-6, 2002.

Watanabe S., Hidaka T., Horiguchi H., Furukawa A., Tsujimoto Y., "Steady analysis of thermodynamic effect of partial cavitation using singularity method", ASME Fluids Engineering Division Summer Meeting and Exhibition, Houston, TX, USA, June 19-23, 2005.

Zwick S. A., Plesset M.S., "On the dynamics of small vapour bubbles in liquids", J. of Mathematical Physics, vol. 33, 1955, p. 308-330. 